\title{
Suicide ideation, stability of symptoms and effects of aerobic exercise in major depression
}

\author{
Andrea Schmitt • Peter Falkai
}

Published online: 24 August 2014

(C) Springer-Verlag Berlin Heidelberg 2014

Suicide is one of the severest consequences of major psychiatric disorders. Among patients with depression, risk factors like duration of disease, living alone, younger age, childhood trauma and previous suicidality have been reported. In a 30-year prospectively followed-up population sample of 4.547 subjects, Angst et al. [1] found a $40.5 \%$ lifetime prevalence rate of suicide ideation and a $6.6 \%$ rate of suicide attempts. Suicide attempts occurred more frequently in females than in males. Main risk factors for suicidal ideation in women were low social support, frequent punishment in childhood, whereas in men, a depressive and anxious personality was predominant. Major risk factors for suicide attempts in women were a broken home and sexual abuse in childhood. Furthermore, in major depression, suicide and suicide attempts are symptoms of relapse. According to the literature, relapse rates vary between 40 and $85 \%$. In a prospective multicenter study of 458 naturalistically treated inpatients with major depression, Seemüller et al. [2] found at least one severe relapse in $33.6 \%$ of the patients during the threeyear follow-up. A shorter duration until severe relapse was associated with multiple hospitalizations, avoidant personality disorder, continuing antipsychotic medication, and lack of antidepressant treatment. In their longitudinal Zurich study, Rodgers et al. [3] investigated the role of sex on stability and transition patterns of depression symptom subtypes over 20 years in 322 patients. This is the first study showing that—based on psychosocial correlates-

A. Schmitt $(\bowtie) \cdot$ P. Falkai

Department of Psychiatry and Psychotherapy, Ludwig-

Maximilians-University Munich, Nußbaumstr. 7, 80336 Munich, Germany

e-mail: andrea.schmitt@med.uni-muenchen.de long-term stability and transition patterns differ by sex and depression subtypes.

As a new therapeutic approach in severe psychiatric disorders, Oertel-Knöchel et al. [4] compared aerobic exercise in patients with major depression and patients with schizophrenia. Physical activity increased cognitive performance in both patient groups, while patients with major depression showed a reduction in depressive symptoms and state anxiety after the intervention period. Aerobic exercise may contribute to modern multi-professional treatment approaches and involve only low side effects. Studies investigating longer intervention periods are needed to assess stable symptom reduction. Although the underlying neurobiological mechanisms are poorly understood, from animal models, it is known that exercise induces neurogenesis and alters the oxidative stress system. Schuch et al. [5] investigated thiobarbituric acid-reactive substances (TBRAS) and brain-derived neurotrophic factor (BDNF) in serum of severely depressed inpatients at baseline and after aerobic exercise. After adjusting for previous tobacco use, exercise decreased TBARS serum levels compared to nonexercising controls, while no effects could be detected for BDNF levels. According to the literature, physical exercise may also influence the immune system.

Krause et al. [6] investigated the role of the innate and adaptive immune response in the development of postpartum depression during pregnancy and postpartum period. They found regulatory T-cells to be increased pre- and postnatally in mothers with postpartum depressive symptoms. Other immune parameters such as postnatally decreased CXCR1 or prenatally increased neopterin levels were associated with postnatal depression. These results may form the basis to develop a biomarker set predicting postnatal depressive symptoms. In addition to the proposed immune markers, a glutamatergic dysfunction has been 
assumed to play a role in the pathophysiology of major depression. AMPA receptors are known to modulate glutamatergic $N$-methyl-D-aspartate (NMDA) receptors and play a role in genesis and treatment of depression. In an animal model, Vogt et al. [7] investigated the effect of temporally and spatially restricted gene manipulation of GluA1, a subunit of the glutamatergic AMPA receptor, on behavioral correlates of mood disorders. In contrast to global AMPA receptor deletion, GluA1 deletion restricted to forebrain glutamatergic neurons during adulthood did not induce a depression- and anxiety-related behavioral phenotype. It is suggested that in the pathophysiology of depression, glutamatergic neurotransmission at AMPA receptors may be disturbed on interneurons and early in development.

Furthermore, major depression is associated with cognitive impairments in semantic verbal fluency. In an fMRI study, Backes et al. [8] examined brain activation during articulation of words in response to semantic category cues. Compared to healthy controls, patients with unipolar depression showed poorer task performance and after correction for task performance, patients displayed reduced task-related deactivation in the right parahippocampal gyrus, right fusiform gyrus and right supplemental motor area. In contrast, an increased activation was observed in the right precentral gyrus and left cerebellum. The results indicate disturbed default-mode network functions accompanied by enhanced recruitment of higher-order language processes as underlying processes of disturbed semantic verbal fluency in major depression. In this patient group, the semantic verbal fluency task may be a sensitive tool for assessing brain dysfunction.

\section{References}

1. Angst J, Hengartner MP, Rogers J, Schnyder U, Steinhausen H-C, Ajdacic-Gross V, Rössler W (2014) Suicidality in the prospective
Zurich study: prevalence, risk factors and gender. Eur Arch Psychiatry Clin Neurosci. doi:10.1007/s00406-014-0500-1

2. Seemüller F, Meier S, Obermeier M, Musil R, Bauer M, Adli M, Kronmüller K, Holsboer F, Brieger P, Laux G, Bender W, Heuser I, Zeiler J, Gaebel W, Riedel M, Falkai P, Möller H-J (2014) Three-year long-term outcome of 458 naturalistically treated inpatients with major depressive episode: severe relapse rates and risk factors. Eur Arch Psychiatry Clin Neurosci. doi:10.1007/ s00406-014-0495-7

3. Rodgers S, Ajdacic-Gross V, Müller M, Hengartner MP, Holtforth MG, Angst J, Rössler W (2013) The role of sex on stability and change of depression symptom subtypes over 20 years: a latent transition analysis. Eur Arch Psychiatry Clin Neurosci. doi:10. 1007/s00406-013-0475-3

4. Oertel-Knöchel V, Mehler P, Thiel C, Steinbrecher K, Malchow B, Tesky V, Ademmer K, Prvulovic D, Banzer W, Zopf Y, Schmitt A, Hänsel F (2014) Effects of aerobic exercise on cognitive performance and individual psychopathology in depressive and schizophrenia patients. Eur Arch Psychiatry Clin Neurosci. doi:10.1007/ s00406-014-0485-9

5. Schuch FB, Vasconcelos-Moreno MP, Borowsky C, Zimmermann AB, Wollenhaupt-Aguiar B, Ferrari P, de Almeida Fleck MP (2014) The effects of exercise on oxidative stress (TBARS) and BDNF in severely depressed inpatients. Eur Arch Psychiatry Clin Neurosci. doi:10.1007/s00406-014-0489-5

6. Krause D, Jobst A, Kirchberg F, Kieper S, Härtl K, Kästner R, Myint A-M, Müller N, Schwarz MJ (2014) Prenatal immunologic predictors of postpartum depressive symptoms: a prospective study for potential diagnostic markers. Eur Arch Psychiatry Clin Neurosci. doi:10.1007/s00406-014-0494-8

7. Vogt MA, Elkin H, Pfeiffer N, Sprengel R, Gass P, Inta D (2014) Impact of adolescent GluA1 AMPA receptor ablation in forebrain excitatory neurons on behavioural correlates of mood disorders. Eur Arch Psychiatry Clin Neurosci. doi:10.1007/s00406-0140509-5

8. Backes H, Dietsche B, Nagels A, Stratmann M, Konrad C, Kircher T, Krug A (2014) Increased neural activity during overt and continuous semantic verbal fluency in major depression: mainly a failure to deactivate. Eur Arch Psychiatry Clin Neurosci. doi:10. 1007/s00406-014-0491-y 